ADP-95-14/T175

March 1995

KFA-IKP(TH)-1995-04

\title{
Mesonic Corrections to the Shape of Quark Distributions
}

F.M. Steffens ${ }^{a}$, H. Holtmann ${ }^{a, b}$ and A.W. Thomas ${ }^{a}$

a) Department of Physics and Mathematical Physics University of Adelaide

Adelaide, S.A. 5005, Australia

b) Institut für Kernphysik, Forschungszentrum Jülich GmbH. 52425 Jülich, Germany

\begin{abstract}
We compute the full $\mathrm{x}$ - dependence of the proton and neutron spin structure functions in the MIT bag model, including the effect of gluon exchange and the meson cloud. Impressive agreement is found for $\mathrm{x}$ larger than 0.1 , where polarised gluons are not expected to play a significant role.
\end{abstract}


At the present moment there is an impressive collection of high energy data on nucleon structure functions that defy a complete understanding. To quote a few, we mention the NMC [1] measurement of the Gottfried sum rule, the NA51 [2] measurement of the sea asymmetry and the (already classical) problem with the polarized structure functions as measured by EMC/SLAC [3 - 6]. In the particular case of the polarized structure functions, it now seems that one has reached the end of the "spin crisis" and the beginning of the spin problem [6, 7]. As it is unlikely that perturbative QCD corrections alone are enough to solve the problem, we should examine the possibility that at least some of the discrepancy arises in the non-perturbative regime. A common approach has been to calculate the desired quantities at some low scale and then evolve them, using the QCD renormalization group equations, to the experimental scale. Unless the model has been specifically derived from QCD, the starting scale at which it best approximates the structure of the nucleon is unknown. It is usually adjusted to fit the experimental parton distributions as well as possible. Of course, one might expect that some models would give a better description of the data than others and there is considerable interest in using DIS to help choose amongst models [8].

Although there are a variety of possibilities to calculate quark distibutions at the starting scale, like non-relativistic quark models [9] or vertex functions [10], here we shall work with the MIT bag. This choice is based on its success in low energy physics, its simplicity and the insights it has already yielded in connecting low and high energy data [11, 12. Of course we do not expect that the bag model alone contains all the physics we need because we know that mesons should play a role as well. For instance, we know [13] that chiral symmetry in the bag is restored through a meson cloud. Moreover, the meson cloud is already known to give important corrections to some sum rules [14 - 19]

As important as it is to correct the integrated quark distributions, the study of the effect of the meson cloud on the $x$ dependence of the quark distributions themselves is at least as important [21 - 24]. In this letter we calculate the $x$ dependence of the polarized and unpolarized quark distributions in the proton in a bag model dressed by mesons. We perform the calculations in next-to-leading order (NLO) QCD. Moreover, we present for the first time the $x$ dependence of the combined effect of the $N-\Delta$ interference term [21] and one gluon exchange [22]. The interference term is important because, as noticed before [14, in the Ellis-Jaffe sum rule it cancels part of 
the reduction coming from pions. As we shall see, the interference terms have a determinant role in the shape of the polarized quark distribution of the neutron.

We introduce mesons in the model through the Sullivan process [25], simply noting that there are unresolved questions about the model, particularly the validity of the impulse approximation [26]. The modern study of the mesonic contribution via convolution started with Thomas 27] and was later extended to the study of structure functions by the Adelaide group [16, 24, 28]. A problem remaining in these calculations is the freedom for the value of the cut-off in the form factor. To avoid this problem, we shall follow the approach of the Jülich group [19, 29], where the cut-off is fixed through the use of high energy $p p$ data.

The basic hypothesis in this sort of model is that the physical nucleon wave function (in the infinite momentum frame) can be written as a superposition of a few Fock states:

$|N\rangle_{\text {phys }}=Z^{1 / 2}\left[|N\rangle_{\text {bare }}+\sum_{B M} \int_{0}^{1} d x \int d^{2} k_{\perp} \phi_{B M}\left(x, k_{\perp}\right)\left|B\left(x, k_{\perp}\right), M\left(1-x,-k_{\perp}\right)\right\rangle\right]$

The wave function renormalization factor

$$
Z=\left[1+\sum_{B M} \int_{0}^{1} d x \int d^{2} k_{\perp}\left|\phi_{B M}\left(x, k_{\perp}\right)\right|^{2}\right]^{-1},
$$

measures the probability that the physical nucleon contains a bare nucleon. The Fock states used in our calculation involve the low mass particles which are important to describe nucleon properties, namely the nucleon $S U(3)$ octet $(N, \Lambda, \Sigma)$ and decuplet $\left(\Delta, \Sigma^{*}\right)$ and the first pseudoscalar and vector meson octet $\left(\pi, K, \rho, \omega, K^{*}\right)$. We have included the hyperon-kaon contributions for completeness, but their actual size is very small $(\sim 2 \%)$ 19, 20, 28.

The Fock state expansion Eq.(11) has consequences for the structure function of the nucleon. Due to the presence of baryon-meson Fock states, the virtual photon can scatter either on the nucleon core or on the meson-baryon system. Formally, the quark distribution $q(x)$ of the nucleon is given by:

$$
q(x)=Z\left[q_{b a r e}^{N}(x)+\sum_{B M}\left(\delta^{M} q(x)+\delta^{B} q(x)\right)\right] .
$$


The contributions from the virtual meson and baryon can be written as the convolution of the meson (baryon) structure function with its longitudinal momentum distribution in the nucleon:

$\delta^{M} q(x)=\int_{x}^{1} f_{M B}(y) q^{M}\left(\frac{x}{y}\right) \frac{d y}{y} \quad$ and $\quad \delta^{B} q(x)=\int_{x}^{1} f_{B M}(y) q^{B}\left(\frac{x}{y}\right) \frac{d y}{y}$,

where $f_{M B}$ and $f_{B M}$ are given by:

$f_{M B}(x)=\int d^{2} k_{\perp}\left|\phi_{B M}\left(1-x,-k_{\perp}\right)\right|^{2}, \quad$ and $\quad f_{B M}(x)=\int d^{2} k_{\perp}\left|\phi_{B M}\left(x, k_{\perp}\right)\right|^{2}$.

In order to conserve charge and momentum, we have the following relation:

$$
f_{B M}(1-y)=f_{M B}(y) .
$$

The functions $f_{B M}(y)$ and $f_{M B}(y)$ can be calculated using time ordered perturbation theory in the infinite momentum frame [28, 29, 31]. The analytic forms for $f_{B M}(y)$ and $f_{M B}(y)$, can be found in Refs. 28, 29.

In practical calculations we need more information, namely the various coupling constants and the vertex form factors $G_{B M}$. The coupling constants can be extracted from scattering experiments and are rather well known [30]. For the vertex form factor we use an exponential parametrization:

$$
G_{B M}=\exp \left[\frac{1}{2 \Lambda_{B M}^{2}}\left(m_{N}^{2}-M_{B M}^{2}\left(y, k_{\perp}^{2}\right)\right)\right],
$$

with $M_{M B}^{2}\left(y, k_{\perp}^{2}\right)=\frac{m_{B}^{2}+k_{\perp}^{2}}{y}+\frac{m_{M}^{2}+k_{\perp}^{2}}{1-y}$. The cut-off parameters $\Lambda_{B M}$ can be estimated, using one boson exchange models, from $n, \Lambda$ and $\Delta^{++}$production in high energy $p p$ scattering (for details see Ref. [19]). They were found to be $\Lambda_{N \pi}=\Lambda_{N \rho}=1.08 \mathrm{GeV}$ and $\Lambda_{\Delta \pi}=\Lambda_{\Delta \rho}=0.98 \mathrm{GeV}$. The procedure used to obtain the cut-off parameters may be questioned because the assumption of single meson exchange being responsible for the process is fairly simple. However, it certainly gives an upper bound for the values of the various $\Lambda^{\prime} s$.

At this point we are still left without the input distributions in equations (3) and (4). For the quark distribution in the pion we use a recent parameterization by Sutton et al. [32]. By using $S U(3)$ symmetry, the quark 
distributions of all the mesons can then be obtained. For the bare quark distribution of the baryons we use the bag model calculation of the Adelaide group [11, 33]. The main advantage of this method is that it ensures energy-momentum conservation and hence the correct support of the quark distributions. It has also been successful in describing data at high momentum transfer, $Q^{2}$, both in leading order [11] and in next-to-leading order [12]. Mesonic corrections lead to further improvement in the predictions of the model and will, among other things, reduce the amount of evolution needed [34]. The form of the quark distribution is given by 111]:

$$
\begin{aligned}
q_{f}^{\uparrow \downarrow}(x) & =\frac{M}{(2 \pi)^{2}} \sum_{m}\left\langle\mu\left|P_{f, m}\right| \mu\right\rangle \\
& \times \int_{\left[M^{2}(1-x)^{2}-M_{n}^{2}\right] / 2 M(1-x)}^{+\infty}\left|\vec{p}_{n}\right| d\left|\vec{p}_{n}\right| \frac{\left|\phi_{2}\left(\vec{p}_{n}\right)\right|^{2}}{\left|\phi_{3}(0)\right|^{2}}\left|\tilde{\psi}_{m}^{\uparrow \downarrow}\left(\vec{p}_{n}\right)\right|^{2} .
\end{aligned}
$$

Here $|\mu\rangle$ is the spin-flavor part of the wave function of the initial state (at rest), $P_{f, m}$ makes the projection onto flavor $f$ and spin projection $m, M_{n}$ is the mass of the intermediate state and $\tilde{\psi}$ the Fourier transform of the quark wavefunction. Equation (8) gives the two quark contribution to the total quark distribution which dominates at intermediate and large $x$. We should also account for the contributions coming from four quarks in the intermediate state, but for simplicity we shall mimic this contribution by a term of the form $(1-x)^{7}$, properly normalized (further discussion on this subject can be found in Ref. [11]).

We use the MRS parametrization [35] of the unpolarized structure functions to fix the parameters of the model; e.g., the radius of the bag, the average mass of the spin scalar $\left(M_{s}\right)$ and spin vector $\left(M_{v}\right)$ diquark in the intermediate state and the low scale, $\mu^{2}$, at which the model is supposed to be valid. In Fig. 11 we show the total valence distribution computed in NLO in the $\overline{M S}$ scheme for the bag dressed with mesons. Very good agreement with the MRS parametrization is found for $\mu^{2}=0.165 \mathrm{GeV}^{2}, R=0.8 \mathrm{fm}$, $M_{s}=0.65 \mathrm{GeV}$ and $M_{v}=0.85 \mathrm{GeV}$. For comparison, we also show the bare bag calculated with the same set of parameters. The bonus of the NLO fit is that it provides a decrease of $\alpha_{s}$ at $\mu^{2}$ from $\sim 1.52$ in a LO fit to $\sim 0.6$ in NLO. This is also a drop in comparison with the value $\alpha_{s} \sim 0.77$ found in

\footnotetext{
${ }^{1}$ Details for $N L O$ calculation in the bag can be found in 12
} 
a NLO fit without mesons [12]. For completeness, we note that these values of the coupling constant were found using $\Lambda_{Q C D}=0.2 \mathrm{GeV}$ and three active flavors.

After we have fixed all parameters in the unpolarized deep inelastic scattering sector, we can explore the consequences for the polarized sector. The calculation of effects due to the presence of higher $B M$ Fock states for the $g_{1}(x)$ structure function is similar to those for $q(x)$ given in Eq.(3). The contribution from the scattering on the recoil baryon is

$$
\delta^{B} \Delta q(x)=\sum_{M} \int_{x}^{1} d_{B M}(y) \Delta q^{B}\left(\frac{x}{y}\right) \frac{d y}{y},
$$

where $d_{B}(y)$ is the polarized, longitudinal momentum distribution. It can be calculated using the same techniques as for the unpolarized case (for details see [19]). The mesonic contribution vanishes because of the pseudoscalar character of the pion. The main difference from the unpolarized structure function is the presence of the $N-\Delta$ interference term [21], which can also be written as a convolution [29]:

$$
\delta^{i n t} \Delta q(x)=\int_{x}^{1} d_{i n t}(y) \Delta q^{N \Delta}\left(\frac{x}{y}\right) \frac{d y}{y} .
$$

The necessary polarized splitting functions $d_{B M}$ and $d_{i n t}$ can be found in the Appendix.

Combining all these contributions, $g_{1}$ for the nucleon is given by:

$$
g_{1}^{\text {phys }}=Z\left[g_{1}^{\text {bare }}+\sum_{B M}\left(\delta^{B} g_{1}^{B}+\delta g_{1}^{\text {int }}\right)\right] .
$$

We now apply Eqs. (9) - (11) to calculate $g_{1}(x)$. In the meson sector only the pseudoscalars are included. The vector mesons are omitted because we are not aware of any model to extract their polarized quark distribution functions. Once again we need a model for the bare quark distributions in the nucleon and, as before, we use the MIT bag model. For the polarized case we need to specify the spin-flavor part of the wave function. We shall use the usual $S U(6)$ wave function in which case, for the interference terms, we have:

$$
\left\langle p^{\uparrow}, n^{\uparrow}\left|u^{\uparrow}\right| \Delta^{+, \uparrow}, \Delta^{0, \uparrow}\right\rangle=\left\langle p^{\uparrow}, n^{\uparrow}\left|d^{\downarrow}\right| \Delta^{+, \uparrow}, \Delta^{0, \uparrow}\right\rangle=\frac{\sqrt{2}}{3},
$$




$$
\left\langle p^{\uparrow}, n^{\uparrow}\left|u^{\downarrow}\right| \Delta^{+, \uparrow}, \Delta^{0, \uparrow}\right\rangle=\left\langle p^{\uparrow}, n^{\uparrow}\left|d^{\uparrow}\right| \Delta^{+, \uparrow}, \Delta^{0, \uparrow}\right\rangle=-\frac{\sqrt{2}}{3} .
$$

We notice that in the matrix elements (12) and (13) only mixed symmetric terms contribute and, as a consequence, the intermediate state always forms a spin vector.

The first moment of the polarized structure function for the proton, $g_{1}^{p}(x)$, is expressed in NLO as:

$$
\int_{0}^{1} g_{1}^{p}\left(x, Q^{2}\right) d x=\left(\frac{g_{a}}{12}+\frac{g_{8}}{36}\right)\left(1-\frac{\alpha_{s}\left(Q^{2}\right)}{\pi}\right)+\frac{g_{0}}{9}\left(1-\frac{\alpha_{s}\left(Q^{2}\right)}{3 \pi}\right),
$$

with $g_{a}$ and $g_{8}$ nonsinglet distributions and $g_{0}$ a singlet distribution. However, the full singlet anomalous dimensions (for any moment) for polarized scattering in NLO are still not known and, because of that, it is not possible to calculate the $x$ dependence of $g_{0}$ in NLO. Faced with this problem, we decided to take the following two approaches to the evolution of $g_{1}$ :

- In the first approach, case (a), we evolve $g_{a}$ and $g_{8}$ as nonsinglet in NLO and evolve $g_{0}$ as a singlet in LO so that $g_{0}$ does not pick up the $\left(1-\frac{\alpha_{s}\left(Q^{2}\right)}{3 \pi}\right)$ correction and the whole structure function is overestimated.

- In the second approach, case (b), we treat $g_{a}, g_{8}$ and $g_{0}$ as nonsinglet combinations and evolve them in NLO. In this case, $g_{0}$ picks up a correction of the form $\left(1-\frac{\alpha_{s}\left(Q^{2}\right)}{\pi}\right)$ such that the corrections due to the NLO evolution are overstimated and the structure function is underestimated. The actual curve must be somewhere between the two approaches.

In Fig. 2 we show the EMC and earlier SLAC data for $x g_{1}^{p}(x)$ together with the bare bag, the bag plus mesons but without the $N-\Delta$ mixing terms and the bag plus mesons plus mixing terms. We stress that the parameters for the bare bag differ significantly from those used in earlier calculations [11]. The meson cloud lowers the bag model prediction over the entire range of $x$, in accordance with earlier estimates for the Ellis-Jaffe sum rule in the bag [14. This is because some of the spin of the nucleon is carried as angular momentum by the mesons. The actual value of the calculated Ellis-Jaffe sum rule at $10 \mathrm{GeV}^{2}$ drops from 0.209 in the bare bag to $\sim 0.173$ in the bag plus mesons for case (a) and to $\sim 0.169$ for case (b). The actual value of the sum rule in the bag model plus mesons, calculated using Eq. (14) as it stands, is $\sim 0.171$, supporting our claim that the full NLO prediction for the 
$x$ dependence of $g_{1 p}(x)$ in the present model is somewhere between the results for case (a) and (b) of Fig. 2. The inclusion of vector mesons is expected to reduce the value of the sum rule somewhat more.

The $x$ dependence for the model, compared with the SLAC E143 data [6] for $g_{1}^{p}(x)$, is shown in Fig. 3, and is quite impressive. This data was taken at an average $3 \mathrm{GeV}^{2}$ and has smaller error bars than earlier experiments. Comparison with this set is also a good test of our model once we have to move to a different $Q^{2}$. The resulting agreement between theoretical and experimental values for $g_{1}(x)$ is inspiring and provides some confidence in the model. At this value of $Q^{2}$ the calculated value for the Ellis-Jaffe sum rule is $\sim 0.171$ for case $(\mathrm{a})$ and $\sim 0.166$ for case $(\mathrm{b})$.

These results shed light on how the spin in the physical proton is shared, suggesting strongly that mesons are responsible for part of the dilution of the spin. In general, we can say that the agreement between the data and the theoretical calculation is very impressive and that further corrections might well bring the entire curve within experimental errors. For instance, we know that in the axial gauge the axial anomaly is related to the polarized gluon distribution [36]. In this context, we think that a reasonable polarized gluon distribution [37] could bring the curve down in the region $x<0.4$.

We would also like to call attention to the role of the $N-\Delta$ mixing term. As said before, it tends to increase the Ellis-Jaffe sum rule. In fact, if the mixing terms were absent, the new value of the sum rule at $Q^{2}=10 \mathrm{GeV}^{2}$ would be $\sim 0.159$ for case (a) and $\sim 0.155$ for case (b). For the $Q^{2}$ compatible with the SLAC E143 data, these values would be reduced to $\sim 0.158$ for case (a) and to $\sim 0.152$ for case(b). Figures 2 and 3 tell us that the rise of $g_{1}^{p}(x)$ due to the mixing terms is confined to the region $x \leq 0.3$. This is because for the mixing term, the mass of the intermediate state is always $M_{v}$, and then the contribution is isolated at smaller $x$ when compared with the other contributions (similar to the down quark distribution in the bag [11]).

The most interesting effect associated with the mixing terms can be observed in Fig. 4 , where $g_{1}^{n}(x)$ is shown. Although in $g_{1}^{p}(x)$ the effect of the mixing terms in the $x$ distribution is not too dramatic because one is adding a small number to a large number, in $g_{1}^{n}(x)$ the effect is relatively large because one is subtracting from numbers near to zero. In fact, for $g_{1}^{n}(x)$ the mixing terms are essential to give to the theoretical curve the shape of the experimental data as measured by the SLAC E142 [5] experiment. The first moment of the calculated polarized distribution of the neutron turns out to 
be $\sim 0.004$ for case $(\mathrm{a})$ and $\sim-0.003$ for case $(\mathrm{b})$. It is also worth noticing that there is a consistency between the calculation of $g_{1 p}(x)$ and $g_{1 n}(x)$ - the same calculation that fits the unpolarized data also makes a good prediction for both $g_{1 p}(x)$ and $g_{1 n}(x)$.

We would like to thank S. Bass, W. Melnitchouk, G. Piller, A. W. Schreiber and J. Speth for valuable discussions during the development of this work. One of the authors (H.H.) would like to thank the German Academic Exchange Service (DAAD) for financial support for his visit to Adelaide. This work was supported by the Australian Research Council, by the DAAD and by CAPES (Brazil).

\section{A Appendix}

$$
\begin{aligned}
d_{N \pi}(y) & =\frac{g_{N N \pi}^{2}}{16 \pi^{2}} \int_{0}^{\infty} d k_{\perp}^{2} \frac{\mid G_{N \pi}\left(y,\left.k_{\perp}^{2}\right|^{2}\right.}{y^{2}(1-y)} \frac{m_{N}^{2}(1-y)^{2}-k_{\perp}^{2}}{\left[m_{N}^{2}-M_{N \pi}^{2}\left(y, k_{\perp}^{2}\right)\right]^{2}}, \quad \text { (A.1) } \\
d_{\Delta \pi}(y) & =\frac{g_{N \Delta \pi}^{2}}{96 \pi^{2}} \int_{0}^{\infty} d k_{\perp}^{2} \frac{\left|G_{\Delta \pi}\left(y, k_{\perp}^{2}\right)\right|^{2}}{y^{4}(1-y) m_{\Delta}^{2}} \\
& \times \frac{\left.\left[\left(y m_{N}+m_{\Delta}\right)^{2}+k_{\perp}^{2}\right]\left[\left(y^{2} m_{N}^{2}-m_{\Delta}^{2}\right)^{2}+8 y m_{N} m_{\Delta} k_{\perp}^{2}-k_{\perp}^{4}\right]_{\Lambda} .2\right)}{\left[m_{N}^{2}-M_{\Delta \pi}^{2}\left(y, k_{\perp}^{2}\right)\right]^{2}}
\end{aligned}
$$

and

$$
\begin{aligned}
d_{i n t}(y)= & \frac{g_{N \Delta \pi} g_{N N \pi}}{16 \sqrt{6} \pi^{2}} \int_{0}^{\infty} d k_{\perp}^{2} \frac{G_{\Delta \pi}\left(y, k_{\perp}^{2}\right) G_{N \pi}\left(y, k_{\perp}^{2}\right)}{y^{3}(1-y) m_{\Delta}} \\
\times & \left\{\frac{-m_{N}(1-y)\left(y m_{N}+m_{\Delta}\right)^{2}\left(y m_{N}-m_{\Delta}\right)}{\left[m_{N}^{2}-M_{\Delta \pi}^{2}\left(y, k_{\perp}^{2}\right)\right]\left[m_{N}^{2}-M_{N \pi}^{2}\left(y, k_{\perp}^{2}\right)\right]}\right. \\
& \left.+\frac{\left(2 m_{\Delta}^{2}+(3 y-2) m_{N} m_{\Delta}-y m_{N}^{2}\right) k_{\perp}^{2}-k_{\perp}^{4}}{\left[m_{N}^{2}-M_{\Delta \pi}^{2}\left(y, k_{\perp}^{2}\right)\right]\left[m_{N}^{2}-M_{N \pi}^{2}\left(y, k_{\perp}^{2}\right)\right]}\right\} .
\end{aligned}
$$




\section{References}

[1] NMC, P. Amaudruz et al., Phys. Rev. Lett. 66 (1991) 2712.

[2] NA51, A. Baldit et al., Phys. Lett. B 332 (1994) 244.

[3] EMC, J. Ashman et al., Nucl. Phys. B 328 (1989) 1.

[4] SMC, B. Adeva et al., Phys. Lett. B 302 (1993) 533.

[5] SLAC E142, P. L. Anthony et al., Phys. Rev. Lett 71 (1993) 959.

[6] SLAC E143, K. Abe et al., preprint SLAC-PUB-6508.

[7] A. W. Thomas, Invited paper at SPIN '94, Bloomington, September 1994, hep-ph/9410335.

[8] A. W. Thomas, Prog. Part. Nucl. Phys. 20 (1988) 21.

[9] M. Traini, L. Conci and U. Moschella, Nucl. Phys. A554 (1992), 731; C. J. Benesh, T. Goldman and G. J. Stephenson, Jr., Phys. Rev. C48 (1993), 1379; H. J. Weber, U. Virginia preprint (1994).

[10] H. Meyer and P. J. Mulders, Nucl. Phys. A 528 (1991) 589; P. J. Mulders, A. W. Schreiber and H. Meyer, Nucl. Phys. A 549 (1992) 498; W. Melnitchouk, A. W. Schreiber and A. W. Thomas, Phys. Rev. D 49 (1994) 127; W. Melnitchouk and W. Weise, Phys. Lett. B 334 (1994) 275.

[11] A. W. Schreiber, A. W. Thomas and J. T. Londergan, Phys. Rev. D 42 (1990) 2226 ; A. W. Schreiber, A. I. Signal and A. W. Thomas, Phys. Rev. D 44 (1991) 2653.

[12] F. M. Steffens and A. W. Thomas, Adelaide preprint ADP-94-27/T166.

[13] A. W. Thomas, Adv. Nucl. Phys. 13 (1984) 1.

[14] A. W. Schreiber and A. W. Thomas, Phys. Lett. B 215 (1988) 141.

[15] H. Høgassen and F. Myhrer, hep-ph 9501414; Z. Phys. C 48 (1990) 295. 
[16] A. I. Signal, A. W. Schreiber and A. W. Thomas, Mod. Phys. Lett. A 6 (1991) 271.

[17] W. Koepf, E. M. Henley and M. Alberg, University of Washington preprint DOE/ER/40427-08-N94.

[18] A. Szczurek, J. Speth and G. T. Garvey, Nucl. Phys. A 555 (1993) 249.

[19] H. Holtmann, A. Szczurek and J. Speth, Jülich preprint KFA-IKP(TH)1993-33.

[20] A. I. Signal and A. W. Thomas, Phys. Lett. B 191 (1987) 3.

[21] A. W. Schreiber, P. J. Mulders, A. I. Signal and A. W. Thomas, Phys. Rev. D 45 (1992) 3069.

[22] F. E. Close and A. W. Thomas, Phys. Lett. B 212 (1988), 227.

[23] S. Kumano and J. T. Londergan, Phys. Rev. D 44 (1991) 717.

[24] W. Melnitchouk, A. W. Thomas and A. I. Signal, Z. Phys. A 340 (1991) 85.

[25] J. D. Sullivan, Phys. Rev. D 5 (1972) 1732.

[26] R. L. Jaffe, in: Relativistic Dynamics and Quark - Nuclear Physics, eds. M. B. Johnson and A. Pickleseimer (New York, Wiley, 1985), pp. 537.

[27] A. W. Thomas, Phys. Lett. B 126 (1983) 97.

[28] A. W. Thomas and W. Melnitchouk, in: New Frontiers in Nuclear Physics, eds. S. Homma, Y. Akaishi and M. Wada (World Scientific, Singapore, 1993), pp. 41 - 106.

[29] H. Holtmann, A. Szczurek and J. Speth, Jülich preprint KFA-IKP(TH)1994-25.

[30] R. Machleidt, K. Holinde and Ch. Elster, Phys. Rep. 149 (1987) 1.

[31] V. R. Zoller, Z. Phys. C 54 (1992) 425; W. Melnitchouk and A. W. Thomas, Phys. Rev. D 47 (1993) 3794. 
[32] P. J. Sutton, A. D. Martin, R. G. Roberts and W. Stirling, Phys. Rev. D 45 (1992) 2349.

[33] A. I. Signal and A. W. Thomas, Phys. Lett. 211B (1988), 481; Phys. Rev. D40 (1989), 2832.

[34] A. W. Thomas, Nucl. Phys. A 518 (1990) 186.

[35] A. D. Martin, W. J. Stirling and R. G. Roberts, Phys. Lett. B 306 (1993) 145 .

[36] G. Altarelli and G. G. Ross, Phys. Lett. B 212 (1988) 391; R. D. Carlitz, J. C. Collins and A. H. Mueller, Phys. Lett. B 214 (1988) 299; A. V. Efremov, J. Soffer and O. V. Teryaev, Nucl. Phys. B 246 (1990) 97; S. D. Bass, B. L. Ioffe, N. N. Nikolaev and A. W. Thomas, J. Moscow Phys. Soc. 1 (1991) 317; S. D. Bass, Z. Phys. C 60 (1993) 343; S. D. Bass and A. W. Thomas, Prog. Part. Nucl. Phys. 33 (1994) 449.

[37] T. Gehrmann and W. J. Stirling, Durham preprint DTP/94/38 (1994), hep-ph-9406212. 


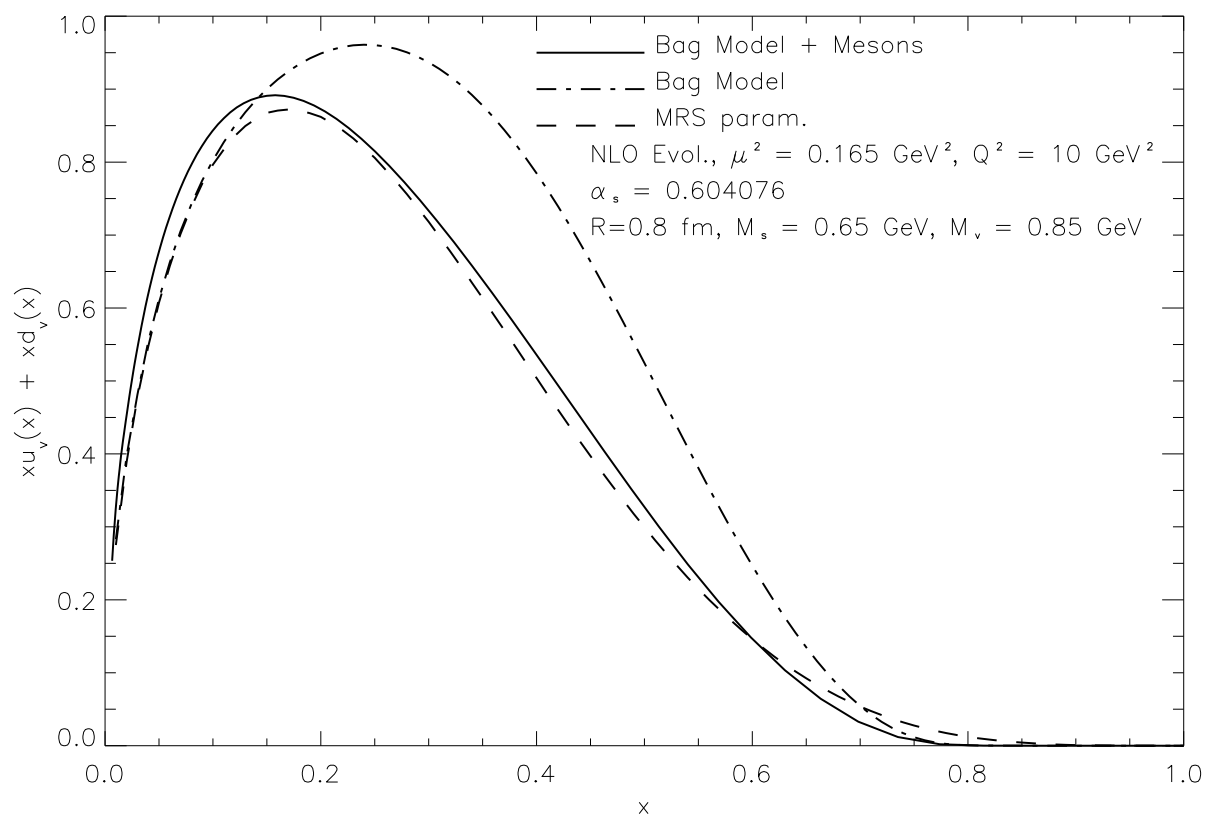

Figure 1: Total valence distribution in the bag and in the bag dressed with mesons compared with the MRS 35] parametrization of the data in the $\overline{M S}$ scheme. The quark distributions are evolved in nest-to-leading-order $Q C D$. 

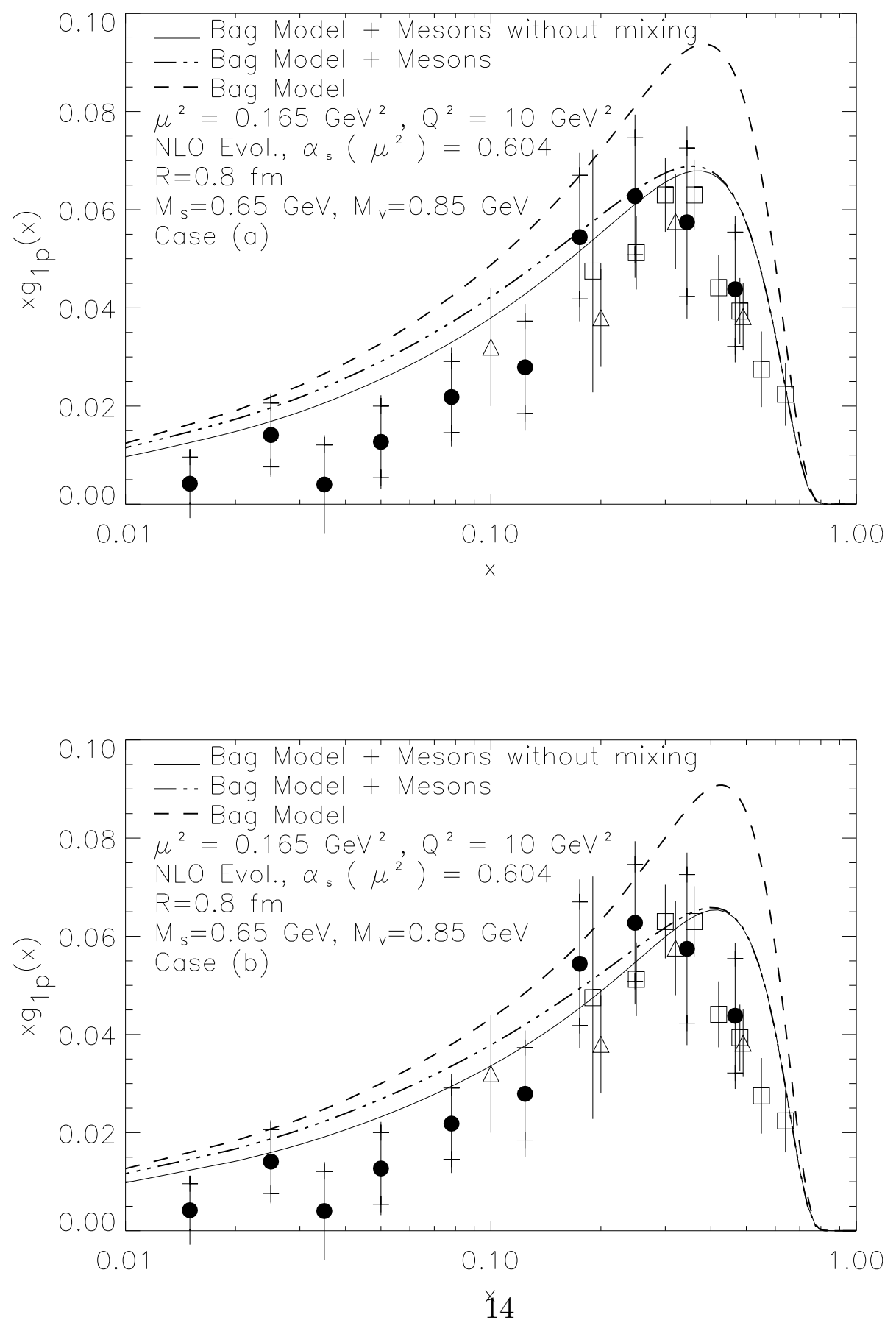

Figure 2: Polarized quark distribution of the proton as measured by the EMC collaboration [3] against theoretical predictions for a bare bag, a bag with mesons without mixing terms and a bag with mesons and mixing terms. 

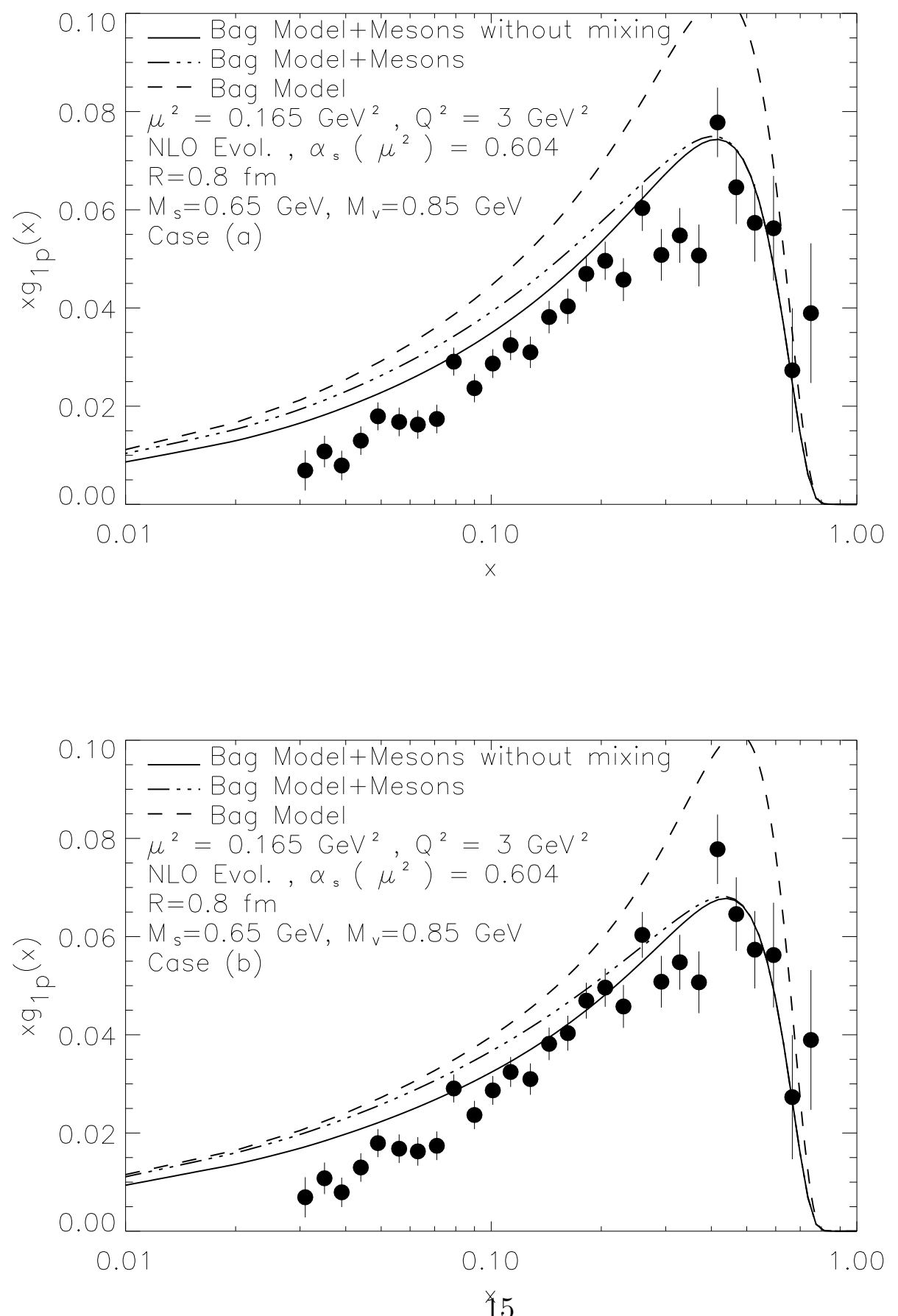

Figure 3: Polarized quark distribution of the proton as measured by the SLAC-E143 experiment [6] against theoretical predictions for a bare bag, a bag with mesons without mixing terms and a bag with mesons and mixing terms. 


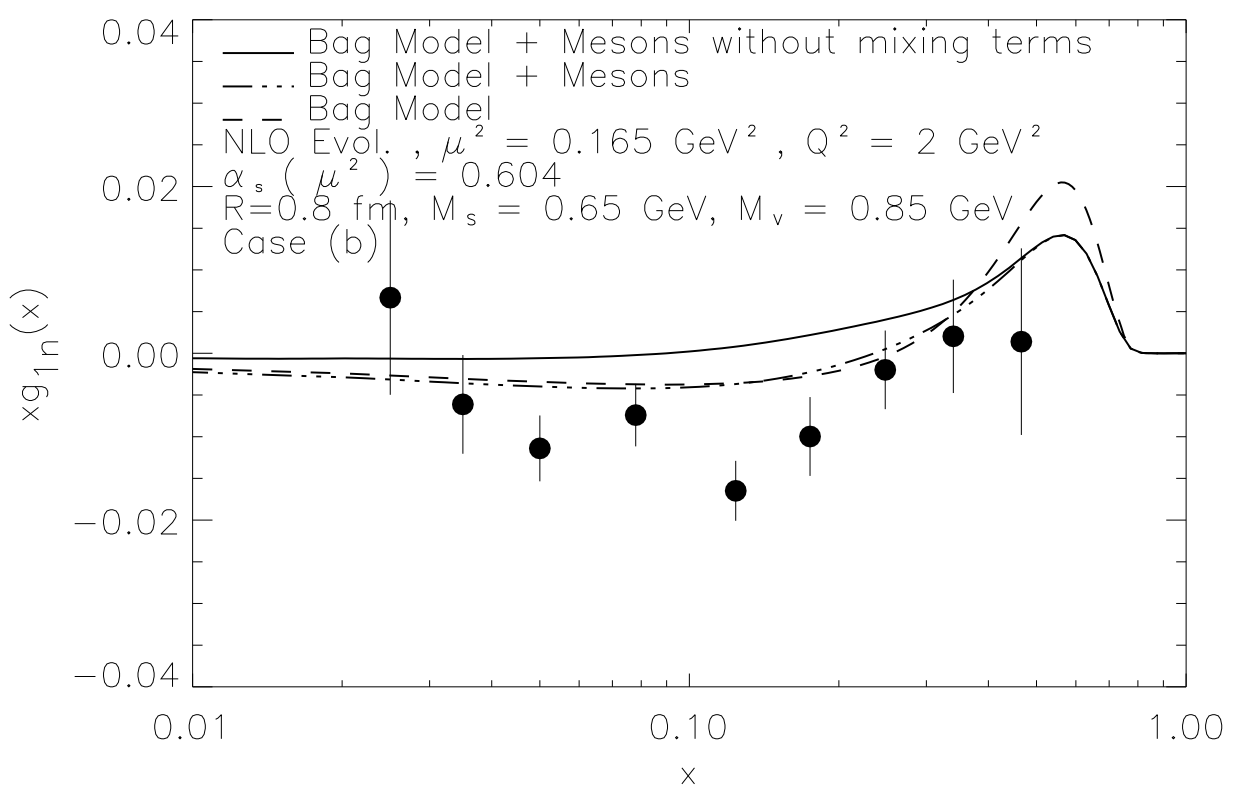

Figure 4: Polarized quark distribution of the neutron as measured by the SLAC-E142 experiment [5] against theoretical predictions for a bare bag, a bag with mesons without mixing terms and a bag with mesons and mixing terms. 